\title{
Mobile microscopy on the move
}

\author{
W.M. Lee ${ }^{\text {a }}$, A Upadhya ${ }^{a}$ and Tri Phan ${ }^{b}$ \\ ${ }^{a}$ Research School of Engineering, College of Engineering and Computer Science, Australian National \\ University, Canberra ACT 0200, Australia \\ ${ }^{\mathrm{b}}$ Garvan Institute of Medical Research, 384 Victoria Street Darlinghurst Sydney NSW 2010 Australia
}

\begin{abstract}
In this paper, we demonstrate the application of low cost light weight imaging device that amplifies the imaging resolution of a smartphone camera by three orders of magnitude from millimeters to sub-micrometers. We attached the lens onto a commercial smartphone camera and imaged micrometer graticules, pathological biological tissue slides and skin which validate the imaging quality of lenses.
\end{abstract}

Keywords: microscope, elastomer optics

\section{INTRODUCTION}

Over the last 400 years, microscopy has transformed from a 17 th century past-time hobby to a striving industry that is forecast to be worth more than US\$ 3.98 billion dollars by 2017 [1]. While the optical technology behind light microscopy have seen little change over the last few centuries [2], the usage of 21st century digital imaging technology in microscopy system has rapidly changed how microscope data are captured and analyzed.

In developing countries, basic light microscopes are severely lacking in developing countries and deny the local population of rapid medical diagnosis of blood borne pathogens and infectious diseases (e.g. malaria parasites, intestinal worms) [3]. In developed countries such as Australia and New Zealand, the occurrence of melanoma, is on the rise. Melanoma makes up only $2.3 \%$ of all skin cancers but is responsible for $75 \%$ of skin cancer deaths [4]. Clinical studies have shown that considerable risks (health and cost) associated with unnecessary biopsy and intervening treatment can be averted using highly mobile epi-luminance microscope [5] that can take high resolution images on the fly.

Existing laboratory microscopes are costly and take up considerable footprint that does not promote mobility, and their handheld counterpart request specialized equipment which lacks generality. Emergence of modern smartphone microscope, in-built smartphone camera with off-the-shelf standard miniature lens elements, has begun to rival its conventional handheld counterpart [6]. In the last few years, there has been a range of mobile smartphone microscope companies such as CellScope from Kholsa Ventures, Lifelens, Keeploop, Handyscope, Proscope etc. The progress of smartphone microscope is part of a global drive to achieve affordable health initiated by multiple telecommunication companies such Qualcomms (US), Vodafone (US, UK) and Telstra (AUS). A research report by research2guidance stated that "The booming mHealth market will grow to $\$ 26$ billion by 2017, reaching a worldwide market of 1.7 billion users looking to use their smartphones and tablets to take care of their health. Currently, there are about 97,000 mobile health applications".

All these factors have motivated the development towards a low cost mobile microscope. A low cost microscope could prove pivotal in a myriad practices in medicine, agriculture, geology, ecology and marine biology, where samples

Digital Photography X, edited by Nitin Sampat, Radka Tezaur, et. al., Proc. of SPIE-IS\&T

Electronic Imaging, SPIE Vol. 9023, 90230A · C 2014 SPIE-IS\&T · CCC code: 0277-786X/14/\$18 doi: $10.1117 / 12.2032754$

Proc. of SPIE-IS\&T/ Vol. 9023 90230A-1 
are examined in microscopic details at anytime and anywhere. A typical scenario would be that a field doctor (i.e. doctors without borders) in a remote village in central Africa can digitally capture hundreds of microscope sample of a blood smears and post the data onto a shared health database. The data can then be scrutinized by a group of medical workers located elsewhere. This will significantly improve accuracy of diagnostic over a large population [5-7].

The performance and cost of any traditional microscope is directly dependent on the properties and fabrication cost of its imaging lenses (Appendix 1). Traditional optical materials are ill-suited for mobile microscope because of rigid material properties and high fabrication cost. Integrating these lenses into smartphone will inevitably increase cost and limits the size of the microscope.

In this paper, we aim to revolutionize the field of mobile microscopy using ultra-lightweight and low cost elastomer microscope lenses. These elastomer lenses are fabricated (mouldless) and tailored to the size of the smartphone imaging sensors. Our microscopes are made up of elastomer lenses [8] and light emitting diode which are lightweight ( $\sim 0.01$ grams) and around $\$ 2$ each to produce. Furthermore, the entire microscope occupied only $\sim 15 \mathrm{~mm}$ in diameter, $\sim 5 \mathrm{~mm}$ thickness. Our microscopes are significantly cheaper and smaller than any existing designs, as shown in see Figure 1.

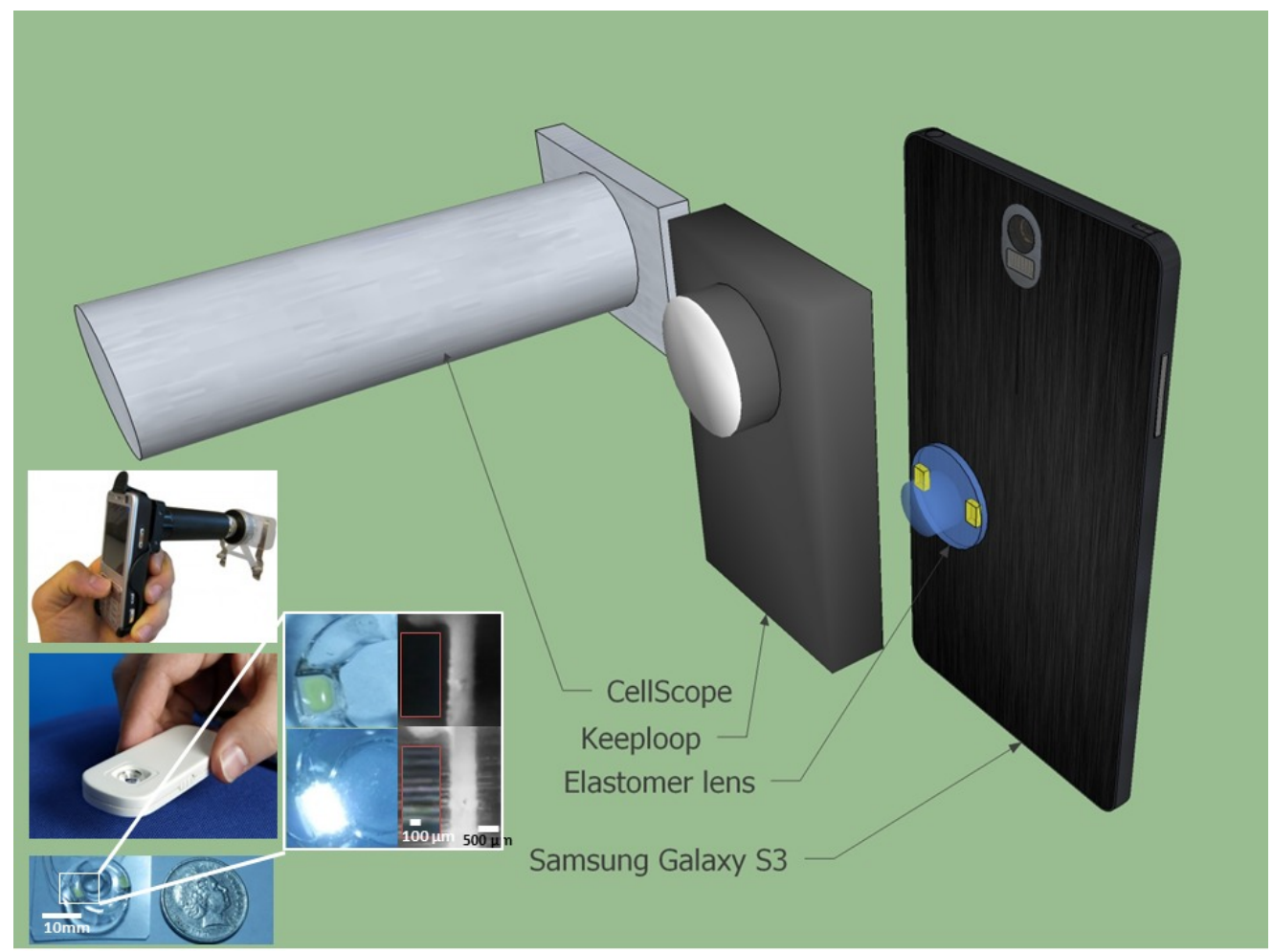

Figure 1 - Size comparison between Mobile microscopes and Samsung Galaxy S3

Cellscope, Keeploop and "Stick-on" microscope with Samsung Galaxy S3 is shown in photographs and 3D schematics. In the inset, LEDs are embedded into elastomer lens (compared to Australian 5 cents coin) being switched on and off with reflectance imaging of engraved metallic patterns. The LEDs are powered by a single coin battery $(1.5 \mathrm{~V}$, diameter $6 \mathrm{~mm})$ 


\section{OPTICAL PERFORMANCE OF ELASTOMER LENSES}

Elastomer optics, patterned in viscous state and solidifies at low temperature $\left(20-80^{\circ} \mathrm{C}\right)$ pioneered by Whiteside and co-workers [8], have shown immense potential as a low-cost material for fabricating high quality imaging and diffractive optics through stamping $[8,9]$. PDMS has flourished in field of microfluidics through the many positive attributions (cost, optical transmission, low birefringence, high surface uniformity, shatterproof, high tensile strength). PDMS optics remains atypical choice for lens-making [8] when compared with polymer and glass because it has lower refractive index (typically $\mathrm{n} 1.4$ ) and physical non-rigidity, A novel fabrication method, that we developed, was shown to yield high powered elastomer lenses. The simplicity in the fabrication technique, as oppose to others, is the significant reduction of manufacturing cost without adversely affecting high resolution imaging ( 10 micrometers and less). The sizes of the rubbers lenses can be made scalable, while maintaining full compatible with smartphones cameras.

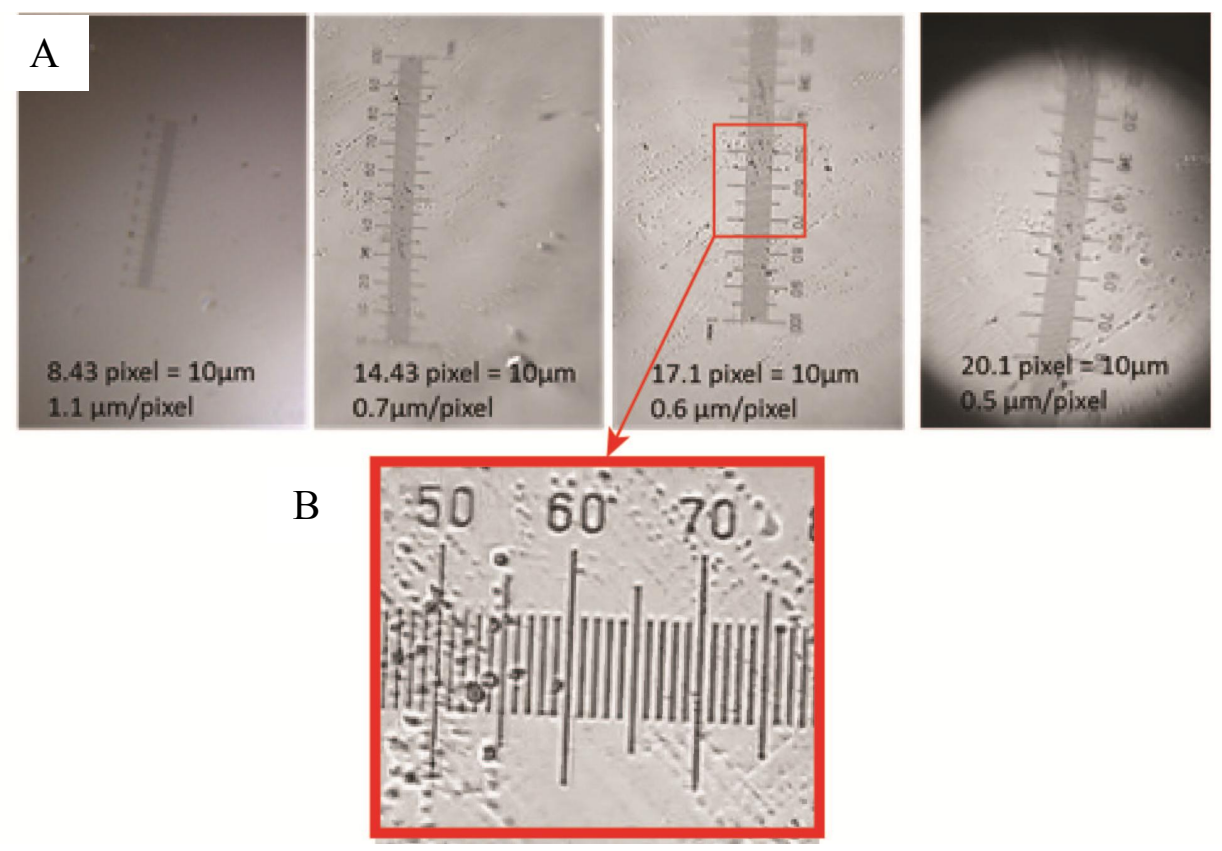

Figure. 2 - Brightfield imaging of microscope graticules (10 micrometer per division). A - 4X digital zoom, B - 16X digital zoom

In figure.2, we describe the imaging performance of the four fabricated lenses of varying focal length using commercially available smartphones in the transmission illumination. A LED light is used as a light source and a microscope scale with binary gratings of around $10 \mu \mathrm{m}$ per division is used as a sample. The lenses are directly attached to the front cover of a 3.15 megapixel smartphone camera (HTC- Explorer) to take the image. The images are digitally recorded using onboard image capture software. In figure. $2 \mathrm{~A}$, the 10 micrometer graticules becomes increasingly visible with lens of shorter focal length with digital zoom (4X) to increase the visibility of the markings. In figure $2 \mathrm{~B}$, we show an image that is digitally zoomed by $16 \mathrm{X}$, where the divisions are clearly resolved. This result showed that the elastomer lenses are capable of resolving down to 10 micrometer that is equivalent to a single cell organism. In practice, the biological samples under examination have much lower contrast. In figure 3, the same light transmission setup is used to 
H\&E stained pathology slides of colon slices (A - D). However, as the biological particles are smaller, we use the lenses on higher resolution camera (8 Mega pixel, Samsung Galaxy 3S). A commercial microscope were used to capture images of the same sample (3B, 3D) and placed alongside for comparison. With the shorter focal length rubber lens (3A, $3 \mathrm{C})$, we can see the markers that have comparable contrast with the bright field imaging of pathology slides taken at 100X (3B) and 40X (3D) respectively. Based on the images taken, we qualitatively conclude that the transmission imaging quality of the elastomer microscope lenses have high enough quality to visualized cell populations on smartphone cameras.
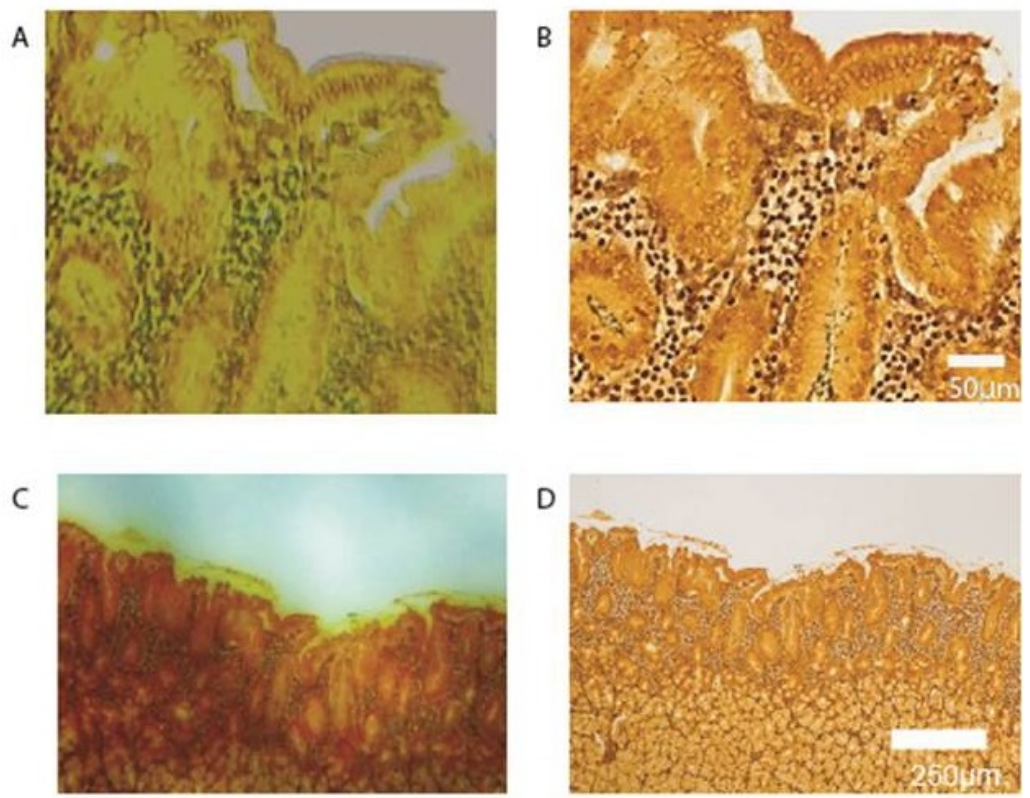

Figure .3 - Pathological slides and dermis imaging performed using Samsung S3 (8Mp) with elastomer lens and Pathology Microscopy. A and C as compared on histological slides of slides of colonic cancer sections taken on pathological grade microscope (B, 40X and D, 100X).

. Next, we examined the imaging quality under reflectance imaging of live biological sample, highly reflective living skin sample. In this imaging trial, we selected the fingertip because of it possess highly undulating features: ridges, sweat pores etc. In figure 4, I can observe these features after minimal image contrast enhancement.

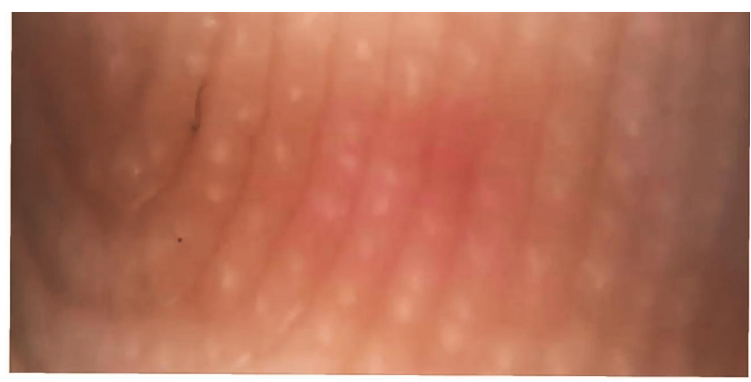

Figure.4 - Reflectance imaging of surface of a fingertip; ridges and sweat pores are visible. 
In practice, larger area and high resolution imaging provide sufficient information for diagnosis. This is achieved by stitching of multiple microscopic images into a single large area. In figure.5, we show that the use of standard stitching software to automatically generate large area image of a LCD display.

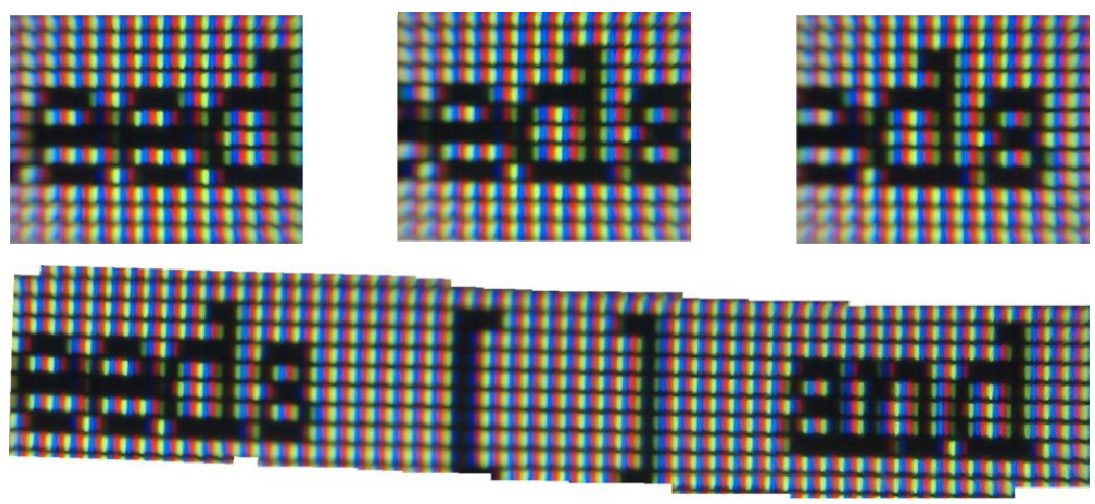

Figure.5 - Stitching of multiple images using Stick-on mobile microscope. Around ten individual microscope images (top- 3 representative images) are sequentially stitched along linear to form a large image mosaic (bottom).

\section{CONCLUSION}

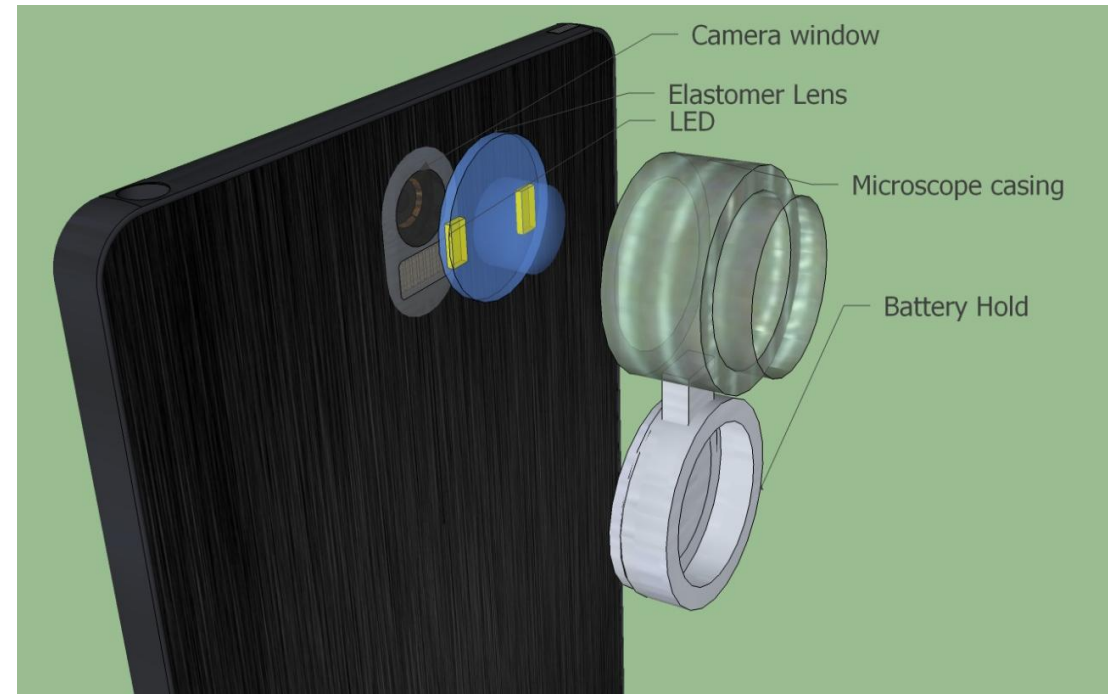

Figure.6 - Prototype design. The image shows breakdown of the individual parts of the microscope. A microscope holder will be fitted with elastomer lens that has embedded LEDs and a battery compartment will be made to house a coin battery.

In conclusion, we conclude that the elastomer lenses increases the imaging resolution of a standard smartphone camera. Using multiple samples ranging from micrometer graticules, biological tissues, the resolution of the lenses have proven to resolve down to single cells $\sim 10$ micrometer in brightfield transmission setup. In figure.6, we show a prototype design of a single smartphone microscope attachment. Finally, though the imaging resolution proved to be 
almost comparable with commercial microscope system, there are still several challenges to overcome The subsequent development of the device will be the resolution of a high volume fabrication process by modifying existing manufacturing platform, increased lenses performance using transparent compensators, stereoscopic imaging (3D like) with controlled oblique illumination, an improved packing design and compatible software based on Android platform. The combination of these factors will not only cement the technical superiority of the microscope and will greatly prepare a rapid manufacturing solution for microscopy masses.

\section{REFERENCES}

[1] Microscopes - global strategic business report, 2011 http://www.strategyr.com/Microscopes_Market_Report.asp

[2] Pawley, James B. "Handbook of biological confocal microscopy" Kluwer Academic Publishers, 1995.

[3] Malaria Facts http://www.cdc.gov/malaria/about/faqs.html

[4] Melanoma facts and statistics http://www.melanoma.org.au/about-melanoma/melanoma-skin-cancer-facts.html

[5] Menzies, S. W., et al. "Impact of dermoscopy and short term sequential digital dermoscopy imaging for the management of pigmented lesions in primary care: a sequential intervention trial." British Journal of Dermatology 161.6 1270-1277 (2009):

[6] Breslauer, David N., et al. "Mobile phone based clinical microscopy for global health applications." PLoS One 4.7 e6320(2009)

[7] Tuijn, Coosje J., et al. "Data and image transfer using mobile phones to strengthen microscopy-based diagnostic services in low and middle income country laboratories." PloS one 6.12 e28348 (2011)

[8] Wilbur, James L., et al. "Elastomeric optics." Chemistry of materials 8.7 1380-1385 (1996)

[9] Anderson, Janelle R., et al. "Fabrication of topologically complex three-dimensional microfluidic systems in PDMS by rapid prototyping." Analytical Chemistry 72,14 3158-3164 (2000) 


\section{Appendix.1 - Comparison of miniature lenses}

\begin{tabular}{|c|c|c|c|c|c|c|c|c|c|}
\hline Lens & $\begin{array}{l}\text { Single } \\
\text { Cell }\end{array}$ & Mold-free & $\begin{array}{l}\text { Elasti } \\
\text { c }\end{array}$ & $\begin{array}{l}\text { Shatter } \\
\text { proof }\end{array}$ & $\begin{array}{l}\text { Scratch } \\
\text { proof }\end{array}$ & $\begin{array}{l}\text { Lateral } \\
\text { Resolut } \\
\text { ion } \\
(\mu \mathrm{m})\end{array}$ & $\begin{array}{l}\text { Weight } \\
\text { (g) }\end{array}$ & Size $(\mathrm{mm})$ & $\begin{array}{l}\text { Cost } \\
\text { (USD\$) }\end{array}$ \\
\hline Graded Index Lens & Yes & No & No & No & No & 1 & $5 g$ & $0.35,1,2$ & $50-70$ \\
\hline Polymer Aspheric & Yes & No & No & Yes & Yes & 1 & $\sim 5 \mathrm{~g}$ & $6,7,10$ & $10-50$ \\
\hline Glass Aspheric & Yes & No & No & No & No & 1 & $\sim 13-20 \mathrm{~g}$ & $6,7,10$ & 300 \\
\hline Elastomer Lens & Yes & Yes & Yes & Yes & Yes & 2.5 & $\sim 0.01 \mathrm{~g}$ & 0.5 to 5 & $\sim 0.002$ \\
\hline
\end{tabular}

Proc. of SPIE-IS\&T/ Vol. 9023 90230A-7 